\title{
A commentary on "Performance measurement and joint production of intended and unintended outputs" by Finn Førsund
}

\author{
Sushama Murty $^{1} \cdot$ R. Robert Russell ${ }^{2}$ \\ Accepted: 19 March 2021 / Published online: 16 April 2021 \\ (C) The Author(s) 2021
}

\section{Overview}

This paper is concerned with issues pertaining to the correct modeling of technologies that generate bad outputs along with good outputs. The author critiques the (Shephard (1970) inspired) approach of Färe et al. $(1986,1989)$ (predicated on the assumption of weak disposability of the pollution-generating technology) and promotes the suitability of the (Frisch (1965) inspired) approach of multiple production relations for modeling pollution.

In the past, we have had the opportunity to read and comment on several of the author's earlier papers on modeling pollution technologies. We are of the opinion that almost all of the issues discussed in this paper are not new: they can be found in these earlier works of the author (notably, Førsund $(2009,2018,1998 / 2016)$ ) or in the publications of others. In particular, tracing the evolution of the author's works over time to this current paper, it seems to us that the approach for modeling emission-generating technologies advocated by the author is converging absolutely to the most primitive case of the by-production approach first proposed by Murty and Russell (2002), empirically implemented by Murty et al. (2012) (hereafter denoted

This is a part of Symposium on Proper modelling of production systems that produce both desirable and undesirable outputs. This symposium has an introduction by Chambers et al. (https://doi.org/10. 1007/s11123-021-00607-y) and is associated with original publication by Førsund et al. (https://doi.org/10.1007/s11123-021-00599-9),

Commentaries by Russell et al. (https://doi.org/10.1007/s11123-02100603), Dakpo et al. (https://doi.org/10.1007/s11123-021-00606-z), Grosskopf et al. (https://doi.org/10.1007/s11123-021-00604-1) and rejoinder by Førsund (https://doi.org/10.1007/s11123-021-00605-0).

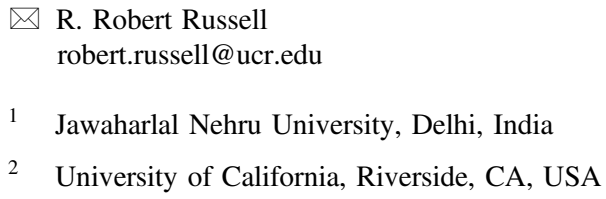

Murty et al. (2012) $)^{1}$, and developed further in a series of papers by (subsets of) the same authors. ${ }^{2}$

The following evaluation focuses on what we consider to be the most important points, including many errors, omissions, and misunderstandings (some of which we have pointed out to the author in past communications). It is organized into six sections (following this overview), sequenced in rough accordance with the substantive sections of the author's paper. The next four sections are relatively anodyne, addressing the author's discussion of the material-balance condition in Section 2, his discussion of the Frisch (1965) models of multi-output technologies in Section 3, and his discussion of models predicated on the Shephard (1970) weak-disposability condition in Section 4. Section 5 points out and corrects some problems with the author's treatment of efficiency and productivity measurement. Our two most important analyses are in Section 6 on the author's treatment of the Murty et al. (2012) byproduction model and in Section 7 on his critique of the treatment of abatement in the by-production framework.

\section{Material balance}

The points mentioned in Section 2 of the paper on material balance seem to be identical to those made by the author in Førsund (2018). Following Baumgärtner and de Swaan Arons (2003), the author explicitly spells out the identities that reflect mass balance for each element forming the inputs and outputs. At the same time, as in Førsund (2018) and Murty and Russell (2020), this paper also appears to take the view that, while in theory the production relations governing emission generation should reflect the two laws

\footnotetext{
${ }^{1}$ Unless the timing is germane, we henceforth refer only to the published paper, Murty et al. (2012), with the understanding that the theory in this paper was substantially developed in the Murty and Russell (2002) discussion paper.

${ }^{2}$ See Murty and Russell (2020) for a survey of this literature, including that on the by-production approach.
} 
of thermodynamics, in practice it might be impossible for the researcher to spell out completely the material-balance conditions. (See the last paragraph of Section 2.)

In the remainder of this section, we take the opportunity to clarify this latter view. This is because, as will be discussed in Section 4 of this commentary, the author seems to ignore this view when critiquing the Shephard-inspired weak-disposability-based approach in his Section 5 and also when critiquing DEA approaches to measuring technical efficiency in Section 6 of his paper. Both of these critiques by the author are based on possible violations of materialbalance conditions by these approaches.

In realistic situations, the way in which emissions are generated depends on several physical factors (often beyond human control). For example, depending on the available oxygen supply, either $\mathrm{CO}$ or $\mathrm{CO}_{2}$ is formed when coal is burned. These two distinct gases have different effects on climate change and the environment. Depending on the temperature at which the chemical reaction occurs, emissions of different oxides of nitrogen are generated. Often, depending on the physical conditions under which chemical reactions take place, new by-products composed of elements not present in the material inputs employed during production are generated because, during the production process, the material inputs react with other elements in the atmosphere. To summarize, all chemical reactions respect the material (mass) balance conditions, but which particular chemical reaction takes place at the time of production might depend on the prevailing physical conditions.

It is also the case that some types of residuals might be omitted from a model of pollution generation because they are relatively harmless and therefore of little relevance to policy making. Hence, although representations of many technologies may appear to be incomplete because they do not explicitly incorporate material-balance conditions, it does not follow that they violate these conditions. Indeed, abstracting from measurement error, observed data on variables modeled by the researcher, when combined with missing or unused data on all unmodeled variables that also figure in the material-balance conditions, must satisfy this physical law.

At the same time, it is important to note that there are instances where data are collected/computed by reporting agencies directly using the material-balance approach. See for instance (i) the pig-finishing farm case in Coelli et al. (2007), in which generation of the bad output $\mathrm{P}_{2} \mathrm{O}_{5}$ attributable to use of two material inputs, piglets and feed, is measured directly using the material-balance approach, and (ii) the thermal power plant case studied in Murty and Nagpal (2019a), Murty and Nagpal (2019b), Murty and Russell (2020), in which the data on $\mathrm{CO}_{2}$ emission are computed by the Central Electricity Authority of India using a linear formula involving emissions and oxidation factors of coal-type and the physical units of coal employed by thermal power plants.

These examples suggest that any good approach to modeling an emission-generating technology should be flexible enough to capture when it exists, the true datagenerating process employed by the data-reporting agencies to compute data on emission generation.

\section{Frisch models of multi-output technologies}

Frisch (1965) first proposed a multi-production relation/ equation approach to modeling production technologies that employ multiple inputs to produce multiple intended/good/ desirable outputs. Førsund (1998/2016) was the first to argue that Frisch's multiple-equation framework is the natural approach to adopt when models of production technologies are extended to incorporate simultaneous production of good and bad outputs. In the current paper, as in almost all of his articles on pollution modeling (e.g., Førsund (1998/ 2016, 2009, 2018)), the author reviews various types of Frisch's production relations and the intuition underlying this relations. (See Section 4 of the current paper.)

We note, however, that all the cases studied by Frisch and reported in the current and previous works of the author are nothing more than suitable applications of the implicit function theorem. This powerful theorem is invoked time and again in economics research in which one solves a system of equations to express some of the variables of the system (often designated as the endogenous variables) as functions of the remaining variables (often called the exogenous variables). ${ }^{3}$

Consider, for example, the case where there are $m$ outputs and $n$ inputs related to each other through $\mu$ equations:

$F^{k}(x, y)=0, k=1, \ldots, \mu$,

where $x \in \mathbf{R}_{+}^{n}$ and $y \in \mathbf{R}_{+}^{m}$ denote an input and an output vector, respectively. If $m=\mu$ and the matrix of first derivatives of the functions, $F^{1}, \ldots, F^{\mu}$, with respect to the output quantities is non-singular, we can solve for the production of each output as a function of all the input levels. This is the case of what the author calls joint production.

If $\mu=1$, then provided the derivative of $F$ with respect to an output is non-zero, we can solve for that output level as a function of all input and remaining output quantities. This

\footnotetext{
3 The implicit function theorem is employed, for example, in comparative static exercises that assess the impact of changes in values of the exogenous variables of the system on the values of the endogenous variables.
} 
corresponds to the case of assorted production with maximum degree of assortment.

Straightforward application of the implicit function theorem similarly yields other possibilities such as product coupling, where $\mu>m$, and factor bands, where $\mu<m$.

\section{Weak disposability}

Arguments similar to those of the author in his Section 5 on how the Shephard-inspired approach to modeling technologies violates the fundamental material-balance condition have been noted in other works in the literature (e.g., Coelli et al. (2007)). ${ }^{4}$ Note however that, as discussed in Section 2 of this commentary, Førsund as well as Murty and Russell (2020) have acknowledged that many valid models of technologies may be incompletely posed owing to, e.g., uncertainties, lack of information, and omission of variables not of interest to the researcher. In such circumstances, it does not make sense to verify whether the material-balance condition holds with respect to the few variables modeled.

The weak-disposability-based literature argues that simultaneous reductions in good and bad outputs are possible along the frontier of the technology when all inputs (including material inputs that cause emissions) are held fixed in the presence of abatement activities undertaken by the firm. The argument is that, as abatement activities draw away the resources of the production unit to mitigate emissions, lesser quantities of both the good and the bad outputs are produced. The critique of this argument provided by the author in Section 5 of his paper seems to draw on that provided in Murty et al. (2012) and Murty and Russell (2020). These authors argue that, since the abatement activities are not explicitly modeled in the weakdisposability-based literature, the model proposed in this literature can be considered to be only a reduced-form representation of the technology. Models employing this approach, however, continue to assume that all inputs, including emission-causing inputs, are freely disposable, which is shown by Murty et al. (2012) and Murty and Russell (2020) to be implausible.

In contrast, while providing his critique of the weakdisposability approach, the author says in the first

\footnotetext{
$\overline{4}$ The author's earlier exposition of the Shephard (1970) weakdisposabiity model in Section 3.1 is confusing and better replaced with a simple reference to Färe et al. (1986). For example, referring to Eq. (3) in his paper, the author states that the "boundary of the sets represents efficient operations". But at this point, his only assumptions are that these sets are closed and the output set is bounded. These are not sufficient to imply efficiency of boundary points. He later introduces the assumptions (4a) and (4b), which partly clarifies the issue, but it remains the case that the points on the vertical segments of the boundaries in Fig. 1, contrary to the author's declaration, are not efficient.
}

paragraph of Section 5 of his paper that it is not possible to draw a diagram like his Fig. 1 in the space of the good and bad outputs, where (i) input levels are held fixed, (ii) inputs are shared between abatement activities and production of good outputs, and (iii) simultaneous reductions in goods and bads are possible along the frontier of the technology. We note however that, as we reduce good and bad outputs along the production frontier in his Fig. 1, it is implicitly assumed that abatement activities are increasing. The figure is explicit in depicting only what happens to good and bad outputs. It is hence not a typical production-possibility set when the firm is also assumed to be engaged in abatement activities. ${ }^{5}$

The main critique of the weak-disposability-based approach, as pointed out by Murty et al. (2012) and Murty and Russell (2020), hence lies in its failure to take into account the fact that disposal of emission-causing inputs is not costless. Ceteris paribus, use of more of these inputs comes at the cost of additional generation of emissions.

Noting some of the above shortcomings of the approach centered on weak disposability, the Shephard-inspired literature has recently been extended to include the assumption of joint-disposability. ${ }^{6}$ This extension implies that emissioncausing inputs are not freely disposable. Rather, such inputs and emissions are assumed to be weakly disposable: it is assumed that equiproportionate reductions in emissions and emission-causing inputs are permitted by the technology. Discussion of this new extension and some of its critiques can be found in Murty and Russell (2018). We feel that this new literature cannot be ignored when discussing Shephardinspired models of emission-generating technologies, as it is an attempt at correcting some of the drawbacks of the conventional Shephard-inspired modeling approaches.

\section{Measurement of efficiency and productivity}

While the author's Section 6.1 on defining technical efficiency contains some worthwhile insights, Section 6.2 on the measurement of efficiency and productivity is flawed and misleading; the author would be well advised to replace this subsection with references to the salient literature.

Owing to the limitation of a single desired output and a single undesired output, the author's formulations of efficiency indexes for the intended-production and emissiongeneration sub-technologies in Eq. (13) essentially ignore all interesting aggregation (index number) problems: each is simply a ratio of two scalar values. Even in this simple

\footnotetext{
5 A typical production possibility set in conventional production theory assumes that all inputs are held fixed.

${ }^{6}$ See, e.g., Ray et al. (2018).
} 
context, the formula is operationally inadequate, since the potential output $y_{i}^{*}$ is a notional concept that should be written as a function of input quantities and the potential pollution level $z_{i}^{*}$, also a notional concept, should be written as a function of pollution-generating input quantities, say $x_{i}^{\prime}$. Of course, this makes the index values themselves functions of the relevant variables, with images $E_{y i}\left(y_{i}, x_{i}\right)$ and $E_{z i}\left(z_{i}, x^{\prime}\right)$.

There are so many problems with the author's formulations of the Malmquist productivity indexes (14) that we hardly know where to begin. The easiest approach is, first, to provide the correct formulation for the intended-output index, put forth by Caves et al. (1982). The Malmquist output-based, technology-t-based productivity index is

$$
M^{t}\left(y^{t}, x^{t}, y^{t+1}, x^{t+1}\right)=\frac{E^{t}\left(y^{t}, x^{t}\right)}{E^{t}\left(y^{t+1}, x^{t+1}\right)}
$$

and the Malmquist output-based, technology- $(t+1)$-based productivity index is

$$
M^{t+1}\left(y^{t}, x^{t}, y^{t+1}, x^{t+1}\right)=\frac{E^{t+1}\left(y^{t}, x^{t}\right)}{E^{t+1}\left(y^{t+1}, x^{t+1}\right)},
$$

where $E^{t}$ and $E^{t+1}$ are the Debreu-Farrell output-based efficiency index mappings for the period- $t$ and period- $(t+1)$ technologies, respectively, and $y^{\tau}$ and $x^{\tau}$ are the output and input quantity vectors (which of course could be scalars as in Førsund's formulations) for periods $\tau=t, t+1$. An analogous construction is formulated for environmental productivity by simple substitution of $z^{\tau}$ for $y^{\tau}$ and $x^{\prime} \tau$ for $x^{\tau}, \tau=t$, $t+1$. Note that these index-number formulations do not require the author's assumption of constant returns to scale.

\section{By-production}

As emphasized in Murty et al. (2012), Murty (2015), and Murty and Russell (2020), the key to correct modeling of an emission-generating technology lies in a proper formulation of its disposability properties. In their widely cited book, Baumol and Oates (1975) ascribe to pollution the standard disposability properties of a conventional input. Murty and Russell (2002), Murty et al. (2012), and Murty (2015) demonstrate that treating emission as a standard input implies that, along the efficient frontier of a technology, the relationship between the emission and each input (including emission-causing inputs) would be negative-a nonsensical implication. ${ }^{7}$

In earlier papers-for example Førsund (1998/2016) Førsund (2009), Førsund (2018)—the author critiqued

\footnotetext{
${ }^{7}$ Recall the negative relationship between two inputs along a standard isoquant, reflecting some degree of input substitutability in producing a given level of a desired/good output.
}

treatment of emissions either as a standard input or as a standard output in terms of the contradictory prescriptions this would yield in the standard welfare maximization problem of a social planner. In Section 3.2 of this paper, however, the author adopts, without attribution, the Murty et al. (2012) critique of the Baumol approach. ${ }^{8}$

The by-production approach of Murty et al. (2012), Murty (2015), and Murty and Russell (2020) distinguishes between emission-generating and non-emission-generating inputs. It models the inevitability of emission generation by noting that the use of every input bundle of emissioncausing inputs is associated with some minimal amount of emission generation. This association identifies the lower (efficient) frontier of emission generation. Along this frontier, emission levels increase as use of emission-causing inputs increases. Murty et al. (2012) showed that these two features of an emission-generating technology imply two disposability properties about emission generation - costly disposability of both emissions and emission-causing inputs: not only are emissions not freely disposable outputs, but disposal of emission-causing inputs also is not free. Along the lower frontier of emission generation, use of more of such inputs comes at a cost: increased generation of emissions.

It was Murty and Russell (2002) and Murty et al. (2012) who first drew a distinction between the lower frontier of the emission-generation technology and the upper frontier of good-output production; the latter identifies the maximal bounds on good-output production given any input bundle. ${ }^{9}$ The author of this paper defines and refers to these two upper and lower frontiers of the technology several times (see e.g., Section 4.2) without citing this earlier work.

In Eq. (11) the author incorporates the constraint $\bar{z} \geq z \geq g\left(x_{M}\right)$, presumably to underscore the existence of an upper bound on emission generation. This bound also has been noted in earlier works (see, e.g., Murty et al. (2012), Murty (2015), and Murty and Russell (2018)). Murty et al. (2012) address this issue in footnote 16: "there, of course, must be an upper bound as well as a lower bound on pollution for given amounts of inputs and intended outputs. We do not incorporate this upper bound into our model because it is only the lower bound that is of interest for policy makers and researchers, and it is only the lower

\footnotetext{
${ }^{8}$ Later, in the first paragraph of Section 4.3 on by-production model, the author acknowledges the Murty et al. (2012) critique, noting that this critique "correspond[s] to the [author's] discussion of Baumol and Oates (1988) in Section 3.2, Eq. (6)". No, the Murty et al. (2012) critique not only corresponds to, but also substantially pre-dates the author's discussion in Section 4.2.

${ }_{9}$ Murty and Russell (2002) explicitly assumed that the pollutiongeneration mechanism, governed by the material-balance condition, is exact and therefore modeled this technology as a manifold. This assumption was relaxed in Murty et al. (2012) to allow inefficiency in pollution generation.
} 
bound that we construct in the numerical examples and empirical application in Sections 4 and 5 below".

The incorporation of an upper (as well as a lower) bound is nevertheless formally modeled by Murty (2015) and Murty and Russell (2018). This formal extension of the Murty et al. (2012) framework entails re-defining $T$ as the free-disposal hull in the direction of the emission variable of the technology in MRL, and showing that the set of (weakly) efficient production vectors in this set are equivalent to the (weakly) efficient production vectors of the true technology set. On the other hand, Førsund's approach to this issue-adding the constraint, $\bar{z} \geq z \geq g\left(x_{M}\right)$-is at best problematic and misleading, since $\bar{z}$, as well as $z$, depends on $x_{M}$, the levels of the material inputs.

Murty et al. (2012), Murty (2015), and Murty and Russell (2020) demonstrate that, in contrast to standard neoclassical technologies, the upper frontier of good-output production and the lower frontier of emission generation are distinct. To illustrate this, consider the case where there is one input and one good output. Here, too, we could derive (i) the upper frontier of the technology as the maximum amount of good-output production for every level of input use and (ii) a lower frontier of the technology as, ceteris paribus, the minimal amount of the input required to produce any given level of good-output production. However, as is well known, in the standard neo-classical case these two frontiers contain the same information; i.e., their graphs are identical. ${ }^{10}$ Either frontier could hence be used to represent a given technology.

Similar arguments are applicable to the case of a neoclassical technology producing multiple outputs. In such a technology, inputs are shared in the production of multiple outputs; i.e., there is rivalry in the use of inputs in producing multiple outputs. ${ }^{11}$ The author, however, states (in footnote 15) that "... if a single transformation function is used with several outputs as arguments, maximizing one intended output at a time keeping the other intended outputs constant for given inputs results in a different production function for each intended output, complicating the usefulness of a single transformation relation".

This statement is false. While several production functions can be defined for a given standard neoclassical technology by maximizing each intended-output production given the levels of other good outputs and inputs, the frontiers defined by these production functions contain the same information: any one of these production functions suffices to obtain a representation of the technology set. A standard neoclassical technology with a single output or

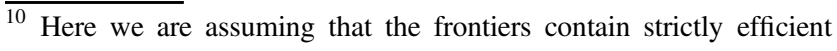
points.

${ }^{11}$ See, also, Murty and Russell (2020).
}

multiple outputs requires only one production function for its representation.

Such conclusions do not hold, however, for all (good and bad) outputs in the by-production specification of Murty et al. (2012). The upper good-output(s) frontier and the lower emission-generation frontier capture different relations between inputs and good and bad outputs. While the lower frontier summarizes the relation between emission generation and use of emission-causing inputs, the upper frontier captures the positive relationships between productive inputs and production of good outputs. The overall technology satisfies both of these types of relationships. This argument underlies the justification for using multiple production functions in the specification of a by-production technology and makes the by-production approach a special case of the work on multioutput production undertaken by Frisch (1965).

To the best of our knowledge, Førsund (1998/ 2016, 2009) was the first to draw attention to the relevance of Frisch's work for modeling emission-generating technologies. The motivation for employing the ideas of Frisch in these works drew on a particular class of multi-output production technologies called joint production, in which inputs are not shared in the production of multiple outputs; rather they jointly produce all the multiple outputs. (See also Murty and Russell (2020) for an analysis of the distinction between joint and rival production.)

The arguments in the earlier papers of the author were not cast, per se, in terms of the disposability assumptions of emissions and emission-causing inputs. Production functions were specified but not the inequalities that specify the full technology. For example, Førsund (1998/2016, 2009, 2018) specifies two production relations of the form,

$y=f\left(x_{M}, x_{S}\right)$ and $z=g\left(x_{M}, x_{S}\right)$,

where $y$ is the scalar output, $z$ is the scalar emission level, $x_{M}$ is the vector of material inputs, and $x_{S}$ is the vector of service inputs. He assumes that $f_{x_{M_{i}}}\left(x_{M}, x_{S}\right)>0$ and $f_{x_{S_{k}}}\left(x_{M}, x_{S}\right)>0$. While in Førsund (2018), $g_{x_{M_{i}}}\left(x_{M}, x_{S}\right)>0$ and $g_{x_{S_{k}}}\left(x_{M}, x_{S}\right)<0$, in Førsund $(1998 / 2016,2009)$, the signs of all the first derivatives of $g$ are unrestricted.

A couple of points need to be noted with respect to specification (1):

(a) It is not clear from this formulation what the functional representation of the technology $T$ is. For example, is it

$$
T=\left\{\langle x, y, z\rangle \mid y \geq f\left(x_{M}, x_{S}\right), z \leq g\left(x_{M}, x_{S}\right)\right\}
$$

or is it

$$
T=\left\{\langle x, y, z\rangle \mid y \leq f\left(x_{M}, x_{S}\right), z \geq g\left(x_{M}, x_{S}\right)\right\}
$$


or is it something else? The precise representation of $T$ is determined by both the monotonicity properties of the functions $f$ and $g$ (i.e., the first partial derivatives of these functions), which determine the trade-offs among inputs and outputs along the graphs of the functions $f$ and $g$ and the frontier of the set $T$, and the disposability properties of $T$. The disposability properties determine which set of these inequalities with respect to the functions $f$ and $g$ define the technology. These disposability properties should be carefully chosen to reflect our real-world observations of the properties of emission-generating technologies: namely, it is costly to dispose of emissions and, along the frontier of the technology, reductions in emissions necessarily entail reductions in the use of emission-causing inputs (abstracting from abatement activities). ${ }^{12}$

(b) As seen in Eq. (1) above, in all earlier works of the author, the arguments of the functions $f$ and $g$ are the same. For example, in Førsund (2018), the presence of service inputs as arguments of the emission-generation function $g$ and the negative signs of its derivatives with respect to service inputs are explained "by the fact that more of a service input improve the utilization of the given raw materials through better process control, fewer rejects, and increased internal recycling of waste materials. The negative partial derivative of service inputs in the residuals function mirrors the positive sign in the output function $f$."

In sharp contrast to the earlier works of the author discussed above, the current paper purges the serviceinput quantity $x_{S}$ from the pollution-generation function $g$. See Eq. (9) in Section 4.2 of the current paper by the author. This is done without explanation and without acknowledgment that this revision brings his model into conformity with the specification of Murty and Russell (2002) and Murty et al. (2012). Moreover, the technology is now represented by inequalities pertaining to the functions $f$ and $g$, implying that the emissiongenerating sub-technology $g$ satisfies assumptions such as costly disposability of emission and emissioncausing material inputs, again bringing the model into conformity with Murty et al. (2012). See Eq. (11) in Section 4.2 of the author's current paper. ${ }^{13}$

\footnotetext{
12 This issue forms the agenda of Murty (2015).

13 In a first draft of this paper, the author acknowledges the source of this change: "Unfortunately, the function $g^{*}($.$) used in Førsund (2018)$ $(\mathrm{a}, \mathrm{b}, \mathrm{c})$ using also $x_{S}$ as an input assuming the marginal product of $x_{S}$ being negative, and the figure illustrating isoquants for both types of outputs in the three publications, are not correct. The relation for the unintended output is correctly specified in Murty et al. (2012); Murty and Russell (2017); Murty and Russell (2018)". This acknowledgment does not appear in his final version.
}

Thus, as seen by the evolution of the author's works over time, his approach to modeling emission-generating technologies is converging to that first proposed by Murty and Russell (2002) and incorporated into Murty et al. (2012). There is, however, a fundamental misconception in Sections 4.2 and 4.3 of his paper. In the concluding Section 7, the author refers to Eq. (11) as "the type of model I have developed in Section 4.2" and refers to Eq. (12) as the byproduction model of Murty et al. (2012) in his Section 4.3. In fact, both (11) and (12) belong to the Murty et al. (2012) class of by-production models. Equation (11) is the pair of constraints defining the by-production technology-stripped down do its bare essentials-while Eq. (12) is the set of constraints defining only the boundary of the by-production technology (with the added feature of abatement activity).

To see this, note that the constraints defining the byproduction model (abstracting from abatement) at page 121 of Murty et al. (2012) $\operatorname{are}^{14}$

$$
F\left(x_{S}, x_{M}, y\right) \leq 0
$$

and

$z \geq g\left(x_{M}\right)$

Invert $F\left(x_{S}, x_{M}, y\right)=0$ in $y$ to obtain $y=f\left(x_{M}, x_{S}\right)$. This converts the above structure to

$y \leq f\left(x_{M}, x_{S}\right)$

$z \geq g\left(x_{M}\right)$

which, apart from the author's problematic upper bound on $z$, is identical to his Eq. (11). There is no difference between the two structures. ${ }^{15}$

One final point regarding the author's attempt to model the by-production model graphically: we cannot make sense of Fig. 2. The main problem is that, while the $y$-isoquants are stationary in this space, the so-called $z$-isoquant would have to shift as the input bundle $\left\langle x_{M}, x_{S}\right\rangle$ moves along either of the isoquants. This confusion reflects the difficulty of trying to encapsulate a three-dimensional problem in a twodimensional graph: multiple graphs, as in Murty and Russell (2020), are needed. In particular, another graph drawn just below Fig. 2 in the space of emissions (on the vertical axis) and $x_{M}$ (on the horizontal axis) would illustrate what

\footnotetext{
${ }^{14}$ Note that precisely this formulation of by-production model (for the special case of one output and two inputs) appears as Eqs. (3.1) and (3.2) in Murty et al. (2012), p. 121.

${ }^{15}$ Note that precisely this formulation of the by-production model (for the special case of one output and two inputs) was presented as such in Russell's discussion of the author's keynote presentation of the paper under review at the EWEPA XVI meeting in London in June 2019.
} 
happens to emission generation as we move along different isoquants in Fig. 2. Reference to $z$ 's in this graph then can be eliminated and captured in the graph below Fig. 2.

\section{Abatement}

The abstract modeling in Murty et al. (2012) is deliberately generic, potentially encompassing many specialized models of particular types of technologies; more restrictive classes of technologies can easily be modeled by the addition of relevant constraints. More information about real-world technologies leads to refined specifications of the model. But all such models satisfying the axioms in Murty et al. (2012) are special cases of the generic byproduction model.

Consider the following comment in the paragraph following Eq. (12) of the current paper: "However, it may be more realistic that the two outputs [intended output $y$ and abatement output $y^{a}$ ] are using different raw materials. Then we may also have two different types of unintended outputs". Of course: this is an example of specializing the model to accommodate particular information. But in no way does this vitiate the general model. An example of such specializations of the model is the specification (10.17) on page 31 of Murty and Russell (2020), which explicitly models the allocation of inputs to desired production and pollution abatement. ${ }^{16}$

Before explicating that specification, however, let us consider a special case where each input is dedicated to either standard production or pollution abatement. Partition the input vectors $x_{z}$ and $x_{o}$ into $x_{z}=\left\langle x_{z}^{Y}, x_{z}^{A}\right\rangle$ and $x_{o}=\left\langle x_{o}^{Y}, x_{o}^{A}\right\rangle$, where superscripts indicate the sub-technology, goods production $(Y)$ or abatement $(A)$, in which the inputs are employed. ${ }^{17}$ In this case, the technology $T_{1}$ is given quite straightforwardly by

$T_{1}:=\left\{\left\langle x_{z}, x_{o}, a, y, z\right\rangle \in \mathbf{R}_{+}^{t} \mid\left\langle x_{z}^{Y}, x_{o}^{Y}, y\right\rangle \in T_{1}^{Y} \wedge\left\langle x_{z}^{A}, x_{o}^{A}, a\right\rangle \in T_{1}^{A}\right\}$,

where $T_{1}^{Y}$ and $T_{1}^{A}$ are production and abatement subtechnologies restricted to the relevant subspaces of the ambient space of the overall technology $T_{1}$.

The (more general) intended-output technology in Murty and Russell (2018) allows for the possibility that each input (whether emission generating or not) is employed in each

\footnotetext{
$\overline{16}$ The author's critique of abatement in the research of Murty and Russell focuses solely on the admittedly spare model in Murty et al. (2012), ignoring the more detailed modeling in Murty and Russell (2020).

17 The two vectors on the right-hand sides of these identities live in subspaces of $\mathbf{R}^{n_{z}}$ and $\mathbf{R}^{n_{o}}$. To keep the notation manageable, we do not formalize this containment feature.
}

sub-technology $T_{1}^{Y}$ or $T_{1}^{A}$. It is defined by ${ }^{18}$

$$
\begin{gathered}
T_{1}:=\left\{\left\langle x_{z}, x_{o}, a, y, z\right\rangle \in \mathbf{R}_{+}^{t} \mid \exists\left\langle x_{z}^{Y}, x_{o}^{Y}\right\rangle \in \mathbf{R}_{+}^{n_{+}+n_{o}} \text { and }\left\langle x_{z}^{A}, x_{o}^{A}\right\rangle \in \mathbf{R}_{+}^{n_{+}+n_{o}}\right. \\
\text { such that } x_{z}^{Y}+x_{z}^{A}=x_{z}, x_{o}^{Y}+x_{o}^{A}=x_{o}, \\
\left.\left\langle x_{z}^{Y}, x_{o}^{Y}, y\right\rangle \in T_{1}^{Y} \subset \mathbf{R}_{+}^{n_{2}+n_{o}+m} \text {, and }\left\langle x_{z}^{A}, x_{o}^{A}, a\right\rangle \in T_{1}^{A} \subset \mathbf{R}_{+}^{n_{z}+n_{o}+1}\right\} .
\end{gathered}
$$

This formulation also allows for the possibility that some inputs are specialized to one of the two sub-technologies. For example, partition $x_{o}$ into two vectors: $x_{o}=\left\langle x_{o 1}, x_{o 2}\right\rangle$, with the understanding that the inputs $o 1$ are employed in the production of conventional outputs and and the inputs $o 2$ are not. Then $\left\langle x_{z}^{Y}, x_{o 1}^{Y}, x_{o 2}^{Y}, y\right\rangle \in T_{1}^{Y}$ implies that $\left\langle x_{z}^{Y}, x_{o 1}^{Y}, \bar{x}_{o 2}^{Y}, y\right\rangle \in T_{1}^{Y}$ for all values of $\bar{x}_{o 2}^{Y}$. That is, the value of $x_{o 2}^{Y}$ is irrelevant to the determination of whether or not this production sub-vector is contained in $T_{1}^{Y}$. Further, the abatement technology may be employing inputs $o 2$ with a typical production vector in this sub-technology being $\left\langle x_{z}^{A}, x_{o 1}^{A}, x_{o 2}^{A}, a\right\rangle \in T_{1}^{A}$

Of course, if it is the case that the researcher knows that the inputs in $o 2$ are not used in the production of the conventional output, we can write a production bundle in this sub-technology as as $\left\langle x_{z}^{Y}, x_{o 1}^{Y}, y\right\rangle \in \mathbf{R}^{n_{z}+n_{01}+m}$, where $n_{o 1}$ is the dimensionality of the vector $x_{o 1}^{Y}$.

Section 4.4 of the paper under review contains several critiques of the above approach to modeling abatement. We respond to each of these criticisms.

"What I have called internal abatement (called prevention in the literature) is not mentioned, but the role of $y^{a}$ in (11) [actually (11) in the old manuscript and (12) in the new manuscript] appears as prevention". This is false on both counts. First, prevention (substitution of clean inputs for polluting inputs) is implicit in the formulation of even the most primitive model in Section 3 of Murty et al. (2012). Points on the boundary of the production set in Murty et al. (2012) satisfy

$f\left(x_{o}, x_{z}, y, y^{a}\right)=0$

$z=g\left(x_{z}, y^{a}\right)$

where, among other constraints, $f$ is decreasing in the two input quantities $x_{o}$ and $x_{z}$ and increasing in the intended and abatement output quantities $y$ and $a$, and $g$ is increasing in $x_{z}$ and decreasing in $a$. Consider a substitution of nonpolluting inputs for polluting inputs along the boundary. This maintains the first equality but lowers the value of $g\left(z_{z}\right.$, $a$ ) in the second equation, thus lowering pollution $z$. This is internal abatement, pure and simple; abatement activity $y^{a}$ plays no role in this exercise. Rather, lowering pollution through increased abatement activity entails a shift of inputs in the first equation from production of intended output to

\footnotetext{
${ }^{18}$ This definition is admittedly, but necessarily, cumbersome.
} 
generation of abatement activity, which then lowers the level of pollution $z$ in the second equation. ${ }^{19}$

"As far as I know the internal type of abatement in the byproduction model has not been implemented empirically in the literature." In fact, it has been: in the empirical section of their by-production paper, Murty et al. (2012) model substitution among multiple fuels-coal, oil and natural gas.

"... it is not so easy to see that the formulation in (11) [actually (12) in new manuscript] of two types of production functions can be turned into three separate equations as required introducing end-of-pipe abatement proper ..." Not only can this be done, it has been done in Murty and Russell (2018), which is cited by the author. Equations (2) and (3) above, taken from Murty and Russell (2018), have two constraints describing the intended-production function in the case of multiple abatement activities. It is true, but of no concern, that the technologies employ two constraints. These set-theoretic constraints of course can be restated as algebraic constraints. For example the constraints in (2) can be written as $y \leq f^{Y}\left(x_{z}^{Y}, x_{o}^{Y}\right)$, where $f^{Y}$ is the production function representing the boundary of $T_{1}^{Y}$, and $a \leq f^{A}\left(x_{z}^{A}, x_{o}^{A}\right)$, where $f^{A}$ is the functional representation of the boundary of $T_{1}^{A}$.

"In end-of-pipe abatement, primary pollutants are used as inputs. This feature seems to be absent in the abatement specification in Murty et al. (2012), Murty and Russell (2018, 2020)." This is false: the function $f^{A}$ defining the amount of abatement (i.e., reductions in emission produced) also has emission-causing inputs as its arguments. Since gross emissions depend on the use of pollution-generating input quantities, we can write $\bar{z}=\psi\left(x_{z}\right)$, where $\bar{z}$ is the gross quantity of emissions. It follows that net emissions are given by $z=g\left(x_{z}\right.$, a) $:=\psi\left(x_{z}\right)-a$.

\section{Compliance with ethical standards}

Conflict of interest The authors declare no competing interests.

Publisher's note Springer Nature remains neutral with regard to jurisdictional claims in published maps and institutional affiliations.

Open Access This article is licensed under a Creative Commons Attribution 4.0 International License, which permits use, sharing, adaptation, distribution and reproduction in any medium or format, as long as you give appropriate credit to the original author(s) and the source, provide a link to the Creative Commons license, and indicate if changes were made. The images or other third party material in this article are included in the article's Creative Commons license, unless indicated otherwise in a credit line to the material. If material is not included in the article's Creative Commons license and your intended use is not permitted by statutory regulation or exceeds the permitted use, you will need to obtain permission directly from the copyright holder. To view a copy of this license, visit http://creativecommons. org/licenses/by/4.0/.

$\overline{19}$ These comparative static relationships could easily be formalized using the implicit function theorem.

\section{References}

Baumgärtner S, de Swaan Arons J (2003) Necessity and inefficiency in the generation of waste. J Ind Ecol 7:113-123

Baumol WJ, Oates WE $(1975,1988)$ The theory of environmental policy. 1st and 2nd editions. Cambridge University Press

Caves DW, Christensen LR, Diewert WE (1982) The economic theory of index numbers and the measurement of input, output, and productivity. Econometrica 50:1393-1414

Coelli T, Lauwers L, Van Huylenbroeck GV (2007) Environmental efficiency measurement and the materials balance condition. J Prod Anal 28:3-12

Färe R, Grosskopf S, Knox Lovell, CA (1985) The measurement of efficiency of production. Kluwer-Nijhoff, Boston

Färe R, Grosskopf S, Lovell CAK, Pasurka C (1989) Multilateral productivity comparisons when some outputs are undesirable: a non-parametric approach. Rev Econ Stat 75:374-380

Färe R, Grosskopf S, Pasurka C (1986) Effects of relative efficiency in electric power generation due to environmental controls. Resour Energy 8:167-184

Frisch R (1965), Theory of production. Dordrecht, D. Reidel Publishing Company

Førsund F (1998/2016) Pollution modeling and multiple-output production theory, Discussion Paper No 10/2016, D-37/1998, Department of Economics and Social Sciences, Agricultural University of Norway

Førsund F (2009) Good modeling of bad outputs: pollution and multiple-output production. Int Rev Environ Resour Econ 3:1-38

Førsund F (2018) Multi-equation modeling of desirable and undesirable outputs satisfying the material balance. Empir Econ 54:67-99

Murty S (2015) On the properties of an emission-generating technology and its parametric representation. Econ Theory 60:243-282

Murty S, Nagpal, R (2019a) Choice of models for emission-generating technologies and designing technical efficiency improvements. Centre for International Trade and Development, School of International Studies, Jawaharlal Nehru University, Discussion Papers Series, No. DP01901

Murty S, Nagpal, R (2019b) Measuring marginal abatement costs in the Indian thermal power sector: a by-production approach, Centre for International Trade and Development, School of International Studies, Jawaharlal Nehru University, Discussion Papers Series, No. DP01906

Murty S, Nagpal R (2020) Measuring output-based technical efficiency of Indian coal-based thermal power plants: a byproduction approach. Indian Growth Dev Rev 13(1):175-206

Murty S, Russell, R. (2002) On modeling pollution-generating technologies. Department of Economics, University of California, Riverside, Discussion Papers Series, No. 02-14 https:// economics.ucr.edu/papers/papers02/02-14.pdf

Murty S, Russell RR (2018) Modeling emission-generating technologies: reconciliation of axiomatic and by-production approaches. Empir Econ 54:7-30

Murty, S, and Russell, R. R. (2020), Bad outputs, Chapter 12. In: Ray $\mathrm{S}$, Chambers R, Kumbhakar S (Eds), handbook of production economics, vol. 1 (theory). Springer, published online https://doi. org/10.1007/2F978-981-10-3450-3

Murty S, Russell RR, Levkoff SB (2012) On modeling pollutiongenerating technologies. J Environ Econ Manag 64:117-135

Ray SC, Mukherjee K, Venkatesh A (2018) Nonparametric measures of efficiency in the presence of undesirable outputs: a byproduction approach with weak disposability. Empir Econ $54: 31-65$

Shephard RW (1970) Theory of cost and production functions. Princeton University Press 